\title{
REPRESENTATIONS OF $l$-GROUPS BY ALMOST-FINITE QUOTIENT MAPS
}

\author{
DONALD A. CHAMBLESS
}

\begin{abstract}
There are numerous existing methods of embedding an archimedean lattice group into a space of extended-real-valued continuous functions defined on a topological space. In this paper the topological space is constructed using the hull-kernel topology and some prime subgroups of a certain extension of the given group, and the representing functions are described as certain quotient maps. The resulting representation essentially coincides with earlier representations given by B. Vulih and by S. J. Bernau.
\end{abstract}

1. Introduction. There is a rather large literature concerning the general problem of embedding a lattice-ordered algebraic structure into a space of continuous, almost-finite functions defined on a suitable topological space. The concepts of prime subgroup, projector, carrier, component of a weak unit, and polar subgroup are among those considerations which have given rise to the topological spaces which have been variously used in this context, [1], [3], [9]-[17]. In general the spaces used cannot be considered as "canonical", the exception here being the representation of Bernau [3].

Johnson and Kist [10] have shown that many of the existing representation theorems of the above type can be considered as essentially describing embeddings of the lattice-ordered algebraic structure being considered into the lattice of all almost-finite continuous functions defined on some space of prime subgroups with the hull-kernel topology, and they point out the central role of the concept of spectral function in such representations.

It follows from the uniqueness of the representation described by Bernau (Theorem 5 of [3]) that the representation described in [11], [15] is essentially the same as that of [3]. The purpose of this note is to show that these also may be viewed as representations utilizing a certain space of prime subgroups with the hull-kernel topology. Additionally we show that there is a rather natural way to describe the representing functions as certain quotient maps.

Let $H$ be an archimedean $l$-group and let $\left\{x_{\lambda}\right\}_{A}$ be a maximal set of pairwise disjoint strictly positive elements. If $K$ is the completion of

Received by the editors June 2, 1970 .

AMS 1969 subject classifications. Primary 0678, 0680.

Key words and phrases. Representation of archimedean l-groups, almost-finite continuous function, Stone topological space.

Copyright (c) 1971, American Mathematical Society 
the divisible hull of $H$ then each polar subgroup of $K$ is a cardinal summand; in particular this is true of the principal polars $x_{\lambda}^{\prime \prime}$. Hence for each $\lambda \in A$ we have $K=x_{\lambda}^{\prime \prime} \boxplus x_{\lambda}^{\prime}$ and for each $k \in K$ we have the corresponding unique representation $k=k_{\lambda}+k^{\lambda}$. This gives rise to an embedding $k \mapsto\left(\cdots, k_{\lambda}, \cdots\right)$ of $K$ into $G=\prod_{A} x_{\lambda}^{\prime \prime}$ (the large cardinal sum). $G$ is then a complete vector lattice with weak unit $e=\left(\cdots, x_{\lambda}, \cdots\right)$, and the injection of $H$ into $G$ preserves all sups and infs existing in $G$. We proceed to consider the representation of $G$.

Notation. We use, unless otherwise specified, the terminology and notation of [6], [8]. $R$ will denote the totally-ordered group of all real numbers, and $R^{e}$ will denote the two-point compactification of $R$ $\left(R^{e}=R \cup\{ \pm \infty\}\right)$. General references on lattice-ordered algebraic structures are [5], [6], [8].

2. Throughout the remainder of this note $G$ will denote a complete vector lattice with weak unit $e>0$. Let $P(G)$ denote the complete Boolean algebra of polar subgroups of $G$ (for a set $S, S^{\prime}$ will denote the corresponding polar subgroup) and let $X$ denote the Stone representation space of $P(G)$.

A component of the unit $e$ is an element $f$ such that $f \wedge(e-f)=0$. By the completeness of $G$ it follows that each $Q \in P(G)$ is a cardinal summand of $G$ and that there exist components $f_{1}, f_{2}$ of the unit $e$ such that $Q=f_{1}^{\prime \prime}=f_{2}^{\prime}$. For each $x \in X$ define $N_{x}$ to be $U\left\{g^{\prime \prime}: g^{\prime \prime} \in K(x)\right\}$ where $K(x)$ denotes the kernel of the Boolean homomorphism $x$. Then $N_{x}=U\left\{z^{\prime}: z^{\prime \prime} \notin K(x)\right\}$ and so it follows that $N_{x}$ is a minimal prime subgroup of $G[10]$, and that the resulting correspondence $x \leftrightarrow N_{x}$ is a bijection between $X$ and the space of all minimal prime subgroups of $G$ [7]. If $\sigma(x)$ denotes the (unique) value of $e$ containing $N_{x}$ then the resulting correspondence $x \leftrightarrow \sigma(x)$ is a bijection between $X$ and the space $\mathfrak{T}$ of all values of $e$ in $G$ (see Proposition 5 of [2]). Imposing the hull-kernel topology on $\mathfrak{T}$ we have

\section{LEMMA 1. $\sigma$ is a homeomorphism.}

Proof. Let $\theta$ be a closed-open set in $X$. Then $\theta=\left\{x \in X: x\left(h^{\prime \prime}\right)=1\right\}$ for some component $h$ of the unit $e$, and $\sigma(\mathcal{\theta})=\mathfrak{N}_{h}$, the set of all $M \in \mathfrak{N}$ such that $h \notin M . \mathfrak{N}_{h}$ is an open set in $\mathfrak{T}$ and thus $\sigma^{-1}: \mathfrak{T} \rightarrow X$ is continuous (since $X$ has a basis of closed-open sets).

Now $X$ is Hausdorff and $\Re=\Re_{e}$ is compact [10]; thus $\sigma^{-1}$ is a homeomorphism.

COROLlaRY. $\left\{\mathfrak{N}_{h}: h\right.$ is a component of $\left.e\right\}$ is a basis of closed-open sets for the hull-kernel topology on $\mathfrak{T}$. IT is compact, Hausdorff, and extremally disconnected. 
For each $M \in \mathfrak{M}$ let $M^{*}$ denote the smallest $l$-ideal of $G$ properly containing $M$ and let $\rho_{M}$ denote the vector lattice isomorphism of $M^{*} / M$ on to $R$ such that $\rho_{M}(e+M)=1$. For each $g \in G$ let $\gamma(g): \mathfrak{I} \rightarrow R^{e}$ be defined by

$$
\begin{aligned}
\gamma(g)(M) & =+\infty & & \text { if } g+M^{*}>M^{*}, \\
& =\rho_{M}(g+M) & & \text { if } g \in M^{*}, \\
& =-\infty & & \text { if } g+M^{*}<M^{*} .
\end{aligned}
$$

Note that $\gamma(g)(M)=+\infty$ iff $g^{+} \notin M^{*}$.

Let $D(\mathfrak{T})$ denote the space of all continuous functions $f: \mathfrak{T} \rightarrow R^{\boldsymbol{e}}$ which are finite on a dense subset of $\mathscr{T}$ (almost-finite). Then $D(\mathfrak{T C})$ is a complete, laterally-complete vector lattice [15].

Lемма 2. For each $g \in G, \gamma(g)$ is an almost-finite continuous function and hence $\gamma$ maps $G$ into $D(\mathfrak{T T})$.

Proof. For each $r \in R, \gamma(r e)$ is the constant function with range $\{r\}$. To show that $\gamma(g)$ is continuous it suffices to show that $\gamma(g)^{-1}(r,+\infty]$ and $\gamma(g)^{-1}[-\infty, r)$ are open in $\mathscr{T}$ for all $r \in R$. A routine computation shows that these sets are $\mathscr{T}_{(g-r e)}{ }^{+}$and $\mathbb{T}_{(g-r e)^{-}}$, respectively. Hence $\gamma(g)$ is continuous.

Suppose now that $0<g \in G$ and that $0<f$ is a component of $e$ and write $f=f_{1}+f \wedge g, g=g_{1}+f \wedge g$. Since $G$ is archimedean, $\cap \Re(=0$ (if $0<a \in \cap$ Tा then for each $n \geqq 1, e \geqq n(a \wedge e) \bmod$ all values of $e-n(a \wedge e)$ ) and each nonzero element of $G$ has a value in $\mathfrak{T}$ (if $0<g \in G$ has no value in $\Re$ then $g \geqq n(g \wedge e) \bmod M$ for all $n \geqq 0$ and all $M \in \mathscr{N})$. If $f_{1} \neq 0$ and $M \in \mathscr{N}$ is a value of $f_{1}$ then we have $f \notin M$ and $g \in M^{*}$, while if $f_{1}=0$ then $f \leqq g$ and it follows from the archimedean property of $G$ that there exists an $N \in \mathscr{T}$ such that $f \notin N$ and $g \in N^{*}$ (note that since $f$ is a component of $e, f \in M^{*}$ for all $M \in \Re$ ). Therefore $\mathfrak{M}_{f} \Phi \gamma(g)^{-1}(+\infty)$ and so $\gamma(g)$ is almost-finite. And in general, if $h \in G$ then $\gamma(h)^{-1}( \pm \infty)$ is the union of $\gamma\left(h^{+}\right)^{-1}(+\infty)$ and $\gamma\left(h^{-}\right)^{-1}(+\infty)$ and hence is nowhere dense in $\mathfrak{T l}$.

THEOREM. $\gamma$ is a vector lattice isomorphism of $G$ into $D(\mathfrak{T M})$ preserving all sups and infs existing in $G$.

Proof. $\gamma$ maps $G$ into $D(\mathscr{T})$ by Lemma 2 . An element of $D(\mathfrak{T})$ is determined by its restriction to any dense subset, and for $g_{1}, g_{2} \in G$ and $r \in R$ it is obvious that the functions $\gamma\left(g_{1}+r g_{2}\right)$ and $\gamma\left(g_{1}\right)+r \gamma\left(g_{2}\right)$ agree on the dense open set $\delta \equiv \gamma\left(g_{1}\right)^{-1}(R) \cap \gamma\left(g_{2}\right)^{-1}(R)$. Also, if $\gamma(g)=0$ then $g \in \cap$ I $=0$, and hence $\boldsymbol{\gamma}$ is a vector space isomorphism. Moreover, if $g_{1} \wedge g_{2}=0$ and $M \in S$ with $\gamma\left(g_{1}\right)(M) \neq 0$ then $g_{1} \notin M$ implies $g_{2} \in M$ so that $\gamma\left(g_{2}\right)(M)=0$. Hence $\gamma$ is a vector lattice isomorphism. 
It follows easily that $\gamma(G)$ is a dense $l$-subgroup of $D(\mathscr{T})$ ) (in fact, an $l$-ideal) and hence (see Lemma 10 of [4]) $\gamma$ preserves all sups and infs which exist in $G$.

Note. As has been previously mentioned (with reference to the Introduction) it follows that the injection of $H$ into $G$ preserves all sups and infs existing in $H$, and hence $\left.\gamma\right|_{H}$ is an $l$-isomorphism of $H$ into $D(\mathfrak{T})$ which preserves all sups and infs existing in $H . \gamma(H)$ is a large $l$-subgroup of $D(\mathscr{T})$ (i.e., $\gamma(H)$ meets every nonzero $l$-ideal in $D(\mathfrak{T}))$, and hence $\left.\gamma\right|_{H}$ has all the properties mentioned in Theorems 4,5 of $[3]$.

\section{REFERENCES}

1. I. Amemiya, A general spectral theory in semi-ordered linear spaces, J. Fac. Sci. Hokkaido Univ. Ser. I 12 (1953), 111-156. MR 15, 137.

2. B. Banaschewski, On lattice-ordered groups, Fund. Math. 55 (1964), 113-122. MR $29 \# 5930$.

3. S. J. Bernau, Unique representation of Archimedean lattice groups and normal Archimedean lattice rings, Proc. London Math. Soc. (3) 15 (1965), 599-631. MR 32 \#144.

4. - Orthocompletion of lattice groups, Proc. London Math. Soc. (3) 16 (1966), 107-130. MR 32 \#5554.

5. G. Birkhoff, Lattice theory, 3rd ed., Amer. Math. Soc. Colloq. Publ., vol. 25, Amer. Math. Soc., Providence, R.I., 1967. MR 37 \#2638.

6. P. Conrad, Introduction à la théorie des groupes réticulés, Secrétariat mathématique, Paris, 1967. MR 37 \#1289.

7. P. Conrad and D. McAlister, The completion of a lattice-ordered group, J. Austral Math. Soc. 9 (1969), 182-208.

8. L. Fuchs, Partially ordered algebraic systems, Pergamon Press, New York; Addison-Wiley, Reading, Mass., 1963. MR $30 \# 2090$.

9. D. G. Johnson and J. E. Kist, Complemented ideals and extremally disconnected spaces, Arch. Math. 12 (1961), 349-354. MR 26 \#6082.

10. —, Prime ideals in vector lattices, Canad. J. Math. 14 (1962), 517-528. MR 25 \#2010.

11. L. V. Kantorovič, B. Z. Vulih and A. G. Pinsker, Functional analysis in partially ordered spaces, GITTL, Moscow, 1950. (Russian) MR 12, 340.

12. H. Nakano, Modern spectral theory, Maruzen, Tokyo, 1950. MR 12, 419.

13. D. Papert, $A$ representation theory for lattice-groups, Proc. London Math. Soc. (3) 12 (1962), 100-120. MR 24 \#A3217.

14. M. H. Stone, $A$ general theory of spectra. II, Proc. Nat. Acad. Sci. U.S.A. 27 (1941), 83-87. MR 2, 318.

15. B. Z. Vulih, Introduction to the theory of partially ordered spaces, Fizmatgiz, Moscow, 1961; English transl., Noordhoff, Groningen, 1967. MR 24 \#A3494; MR 37 $\# 121$.

16. K. Yosida, On vector lattice with a unit, Proc. Imp. Acad. Tokyo 17 (1941), 121-124. MR 3, 210.

17. —- On the representation of the vector lattice, Proc. Imp. Acad. Tokyo 18 (1942), 339-342. MR 7, 409.

Tulane University, New OrLeans, Louisiana 70118 